\title{
Dynamic Interface Reconfiguration Based on Different Ontological Relations ${ }^{\star}$
}

\author{
Elisa Chiabrando, Roberto Furnari, Pierluigi Grillo, \\ Silvia Likavec, and Ilaria Lombardi \\ Università di Torino, Dipartimento di Informatica, Torino, Italy \\ \{chiabrando,grillo, furnari, likavec, lombardi\}@di.unito.it
}

\begin{abstract}
This paper presents a framework and a prototype implementation of a system that integrates ontological knowledge base with the services for the users in mobility. Our system tries to help users navigate the domain of gastronomy, offering a possibility to explore the ontological base consisting of many concepts and relations. We present the logic and technical realization of the modules responsible for knowledge base interrogation and for presenting the obtained information to a user. We also provide a brief description of the implemented interface and user evaluation.
\end{abstract}

\section{Introduction}

The Internet started as a plain static information source, but, nowadays, it is rapidly changing to a dynamic interactive platform where users actively produce content, create social networks and interact with each other. It became possible to obtain information on the move, in the moment when it is needed, due to increased number of mobile devices on the market and the availability of Internet access in virtually any situation.

Huge amount of information available on the Web is organized in different modes, with ontologies being used very often to model various domains, since they provide structured information on which reasoning is readily available.

Visualizing vast amounts of heterogeneous information in social networks is a challenging task. Especially in the "Mixed social networks", where objects of the domain and users of the network can interact with each other. In our framework, we create exactly this kind of network, enabling the interaction among people and physical objects as indistinguishable parts of the society, as well as the interaction of people with virtual avatars of the objects that continue to exist even when the user is not in direct contact with the physical objects.

In particular, the framework described in this paper is a part of the PIEMONTE Project 9], in which a Social Networking Application in the domain of gastronomy is being developed by integrating social networking and augmented reality tools. In this particular domain, there is a growing need for information

\footnotetext{
* This work has been supported by PIEMONTE Project - People Interaction with Enhanced Multimodal Objects for a New Territory Experience.
} 
about certain products, local producers, production technologies, as well as about similar users and their opinions. Our application developed for Apple iPhone tries to support producers and end-users, meet the expectations of users in mobility and enhance their mutual interaction and overall experience.

We found sophisticated solutions to present the users with different aspects of the domain in a gradual way, enabling them to profit from the great amount of information available. To this aim we developed a new interaction model called the "wheel". The main contributions of our framework are the following:

- a strategy to select the content to be presented to the user (content of the "wheel");

- a novel visualization technique for presenting the relevant information (appearance of the "wheel");

- a novel interaction technique for multi-touch mobile devices (spinning the "wheel").

In this paper we concentrate on the technical realization of the framework and the wheel content selection strategy based on reasoning over domain knowledge base and analyzing the user model. We briefly describe the visualization technique for presenting the information to the user, the details of which can be found in [1].

The paper is organized as follows. In Section 2 we touch upon some related work in the field. We describe the architecture we developed for our framework in Section 3 . followed by the ontological organization of the domain given in Section 4 . In Section 5 we discuss how the relations among the objects of the system and between users and the objects of the system are established. A brief overview of the developed interface is given in Section 6. In Section 7 we summarize the evaluation results and conclude in Section 8

\section{Related Work}

The objective of SEWASIE project 1 is to provide users with uniform access to heterogeneous ontological data. In [3] authors describe the basics of an intelligent user interface used to query such data. Our aim is different, we want to provide users with an opportunity to navigate through a vast space of information in a ludic and intuitive way.

An example of using mobile phones to access ontologically organized data is given in SMARTWEB project 2 In [13] a context-aware, multi-modal mobile application for the Semantic Web is developed. The users interact with the application using smartphones, asking questions using speech and gesture. In [12] the users can obtain answers to their questions in a specific domain as multimodal presentations based on graph visualization and ontological navigation.

Another framework for ontology navigation is presented in [5]. Users can freely explore the ontology or query the information system, neither needing to have the

\footnotetext{
${ }^{1}$ http://www. sewasie.org/

${ }^{2}$ http://www. smartweb-project.de/
} 
knowledge about the internal organization of the data, nor about the vocabulary used to describe the domain. Their software called Query Tool consists of three components: the query logic used to reason over the ontology, the engine for natural language generation, and user interface.

With respect to the mentioned works, although in our framework ontologies play a central role in the domain representation, the ontological knowledge is enriched by knowledge from other sources and offered to the user by means of a Resource Oriented Architecture.

The work in 4 ] presents a hardware kit (electronic bracelet with sensors, RFID reader and accelerometer), which allows the user to interact with real objects. Using hand gestures the user can browse vocal menu provided by objects and select the actions to perform.

11] describes a Physical Mobile Interaction Framework (PMIF) for the communication between a device (e.g. smartphone) and objects in the environment. These objects can communicate with the device "passively" using RFID, NFC, visual markers or bluetooth connection, or they can communicate "actively" having their part of framework-client (PMIF) installed.

On the other hand, in our framework communication with objects is much simpler, since we prefer to use a common device, such as a smartphone, to achieve interaction with objects where there is no need to modify them by inserting codes or installing sensors.

With respect to the software architecture we used, the benefits of using a Resource Oriented Architecture are highlighted in [6] in a Web of Things context; although in a different context, similar consideration can be done in our work, where resources represent a sort of avatars of real things that tell the users about their properties and their relations.

\section{System Design and Implementation}

In the following section we briefly describe the architecture we developed for our framework. Figure 1 depicts the layered architecture of our framework, where client applications ask for particular aspects of domain knowledge and the service infrastructure provides the information required, enabling the clients to browse the domain knowledge gradually and smoothly.

The requested information is provided to the client applications by means of a set of related resources organized in a Resource Oriented Architecture [10]. In such a perspective the items composing the domain knowledge can be easily inquired about their own features and relations with the other domain resources. Following these links between resources it is possible to browse the network of domain items and discover new parts of the domain in a consistent way.

The Resource Oriented Architecture in our framework is managed by means of the Service Logic Layer, the core component of the server side infrastructure, while the Exposure Layer plays the role of a controller that manages the communication between the Service Logic Layer and the client applications from the Application Layer, providing also a monitoring service. 


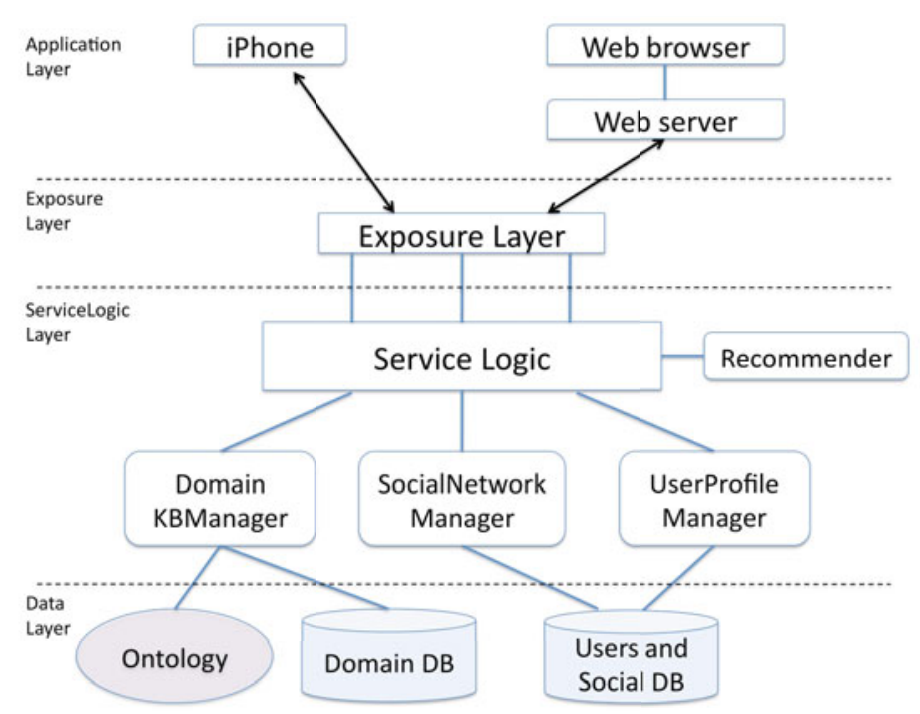

Fig. 1. System architecture

The Service Logic Layer organizes the different knowledge sources present in the Data Layer synthesizing a uniform representation of the resources. When requested, specialized modules (UserProfile Manager and Recommender) are exploited to customize the service depending on the user interests.

In the Data Layer, two main kinds of knowledge exist: a domain knowledge where the domain items and their relations are represented (see Section 4 for more details), and a social knowledge where the information about the users and their social actions is stored. In particular, the DomainKB Manager offers an integrated view of the two data sources used in the Data Layer in order to represent the domain knowledge: the ontology and a database storing a sort of master data set3 about domain items.

The architecture has been realized using Java Environment where the Restlet too 4 and Apache HTTP libraries collaborate in order to answer the HTTP requests from clients. The data are stored using an OWL ontology integrated with a relational MySQL database. The DomainKB Manager uses the D2RQ too 5 to provide a uniform virtual data source that can be directly inquired by means of SPARQL query. Mechanisms of caching are implemented by the Service Logic in order to optimize the request answering process. The information toward a client is serialized in JSON streams, which makes the interoperation

\footnotetext{
${ }^{3}$ By master data set we mean long text description, contact information, pictures and video references, etc. This is all the information about the domain items that does not benefit from the ontological representation since it is excluded from the inference reasoning.

${ }^{4}$ http://www.restlet.org/

${ }^{5}$ D2RQ allows to view a part of a relational database as a set of OWL individuals.
} 
with different client types smooth and seamless. In fact, although the focus of this paper is on the Apple iPhone mobile client, different clients like Web Servers are also served by this architecture. Our application for Apple iPhone has its complement in a form of a web application that provides the producers with the opportunity to enter the gastronomical world and make their products known.

\section{Ontological Organization of the Domain}

Our framework uses ontologies to represent the domain knowledge as a machine recognizable content. Adopting ontologies as knowledge bases enables smooth description and integration of data, as well as queries and reasoning on them. Ontologies describe the domain items by expressly representing their properties and the relations among them, highlighting their similarities and differences. Ontology tools, such as subclasses and restrictions, provide a well structured and organized description of the domain where new data can be seamlessly added. Moreover, ontology reasoning makes available, by means of inference, properties and information that are implicit in the knowledge base. In this project we use OWL 26 - the Web Ontology Language, that extends the previous OWL definition with support for datatypes with ranges and metamodeling.

The domain of our application is gastronomy where we deal with different kinds of domain items such as products, actors related to products (producers, farms, restaurants), as well as recipes and geographical data. The entities of the domain are modeled as elements of the ontology containing very general classes such as Wine or Cheese, as well as very specific ones, such as Dolcetto d'Asti di Vittorio Ubertone or Toma di Lanzo di Marino Dardino.

In order to cover this wide range of knowledge, the model we use is composed of several ontologies, specifying different domain parts, that are imported by the global upper ontology. In particular, we use the following ontologies:

- Taste Ontology is the global upper ontology which imports the other ontologies and where the connections between objects belonging to different ontologies are made explicit.

- Products Ontology is the most populated ontology which describes the products of the domain. It includes other sub-ontologies that detail particular product branches. For example, the Wine Ontology and Cheese Ontology classify and describe wines and cheeses in great detail. Items are grouped by kinds and properties related to their organoleptic features, production procedures and membership in particular quality consortiums.

Furthermore, a set of SWRL 7 rules are defined on this ontology to relate foods based on their properties: in particular, associations between wines and cheeses are defined.

\footnotetext{
${ }^{6}$ http://www.w3.org/TR/ow12-overview/

${ }^{7}$ http://www . w3.org/Submission/SWRL/
} 
- Actors Ontology stores the actors playing active roles in our domain. Both virtual actors, such as food producing companies, farms, restaurants or shops, as well as physical actors, such as producers, restaurant chefs and sellers are present. The actors are related to products by properties: producers produce products, cooks and restaurants use them etc.

- Geo Ontology is a taxonomy of the geographical places of the domain (places of origin of products, places where the products are sold etc.). It includes the administrative regions, as well as the areas that do not correspond to administrative divisions but are widely used to identify particular places. For instance valleys like Valsusa or Val di Lanzo are the names referring to the regions included in the district of Turin and grouping several municipalities.

- Recipes Ontology with the ingredients for each recipe, together with the cooking instructions. Note that the ingredients of a recipe can be recipes itself (e.g sauces or creams), which makes the relationship among ontology elements particularly interesting.

As mentioned in Section 3 the domain knowledge, provided by ontologies, is enriched with the information from the data base. This results in a complete domain description where further investigations about relations among domain items take place.

\section{$5 \quad$ Inferring Object Relations}

Our framework, like other Social Web applications, encourages users to provide content, such as new entities of the domain, tags, ratings and comments. Since this causes a considerable growth of the available information which risks to overwhelm the user, a strategy to limit and tailor the information presented to the user is of major importance.

Our Recommender module is in charge of adapting the content presented to the user by filtering and, most notably, ranking the entities, taking into account user preferences and the relations among entities (objects and people) of the system. Such relations, which are an essential part of the mixed social network, are described in the ontology or can arise from users' actions.

The most natural way to define a link between two instances in the ontology is by using the ObjectProperty. For example, the class Product can have an ObjectProperty producedBy whose range is the class Producer. Moreover, relations between entities and places, or between products and actors in the production chain, as well as restaurants, shops, recipes and chefs exist. We also define SWRL rules to express more sophisticated associations based on the property values of the instances. For example, a set of rules pairs cheeses and wines to be served together, considering their organoleptic properties.

An interesting aspect of the system is that it can infer relations based on user actions. Apart from explicit associations, for example, if a user chooses another person as a friend or if she adds a product as a favorite, the system can recognize implicit connections analyzing tags and comments. Hence two entities 
can be linked if one is tagged with the name of the other one, or if they are tagged with the same label by a significant number of users.

This kind of analysis allows for dynamic inferring of user preferences and of users' similarity. On the one hand, the actions the user performs on entities are claims of interest on them, with an increasing degree from rating, to tagging and commenting. On the other hand, the system can infer a relationship between two users who perform similar actions on the same objects as this reveals a similarity between them.

\section{Content Presentation}

As stated above, the interaction with the user is carried out using a mobile device (Apple iPhone) where the user can explore the domain content by using the WantEat application. WantEat is an application which unifies the default elements of an iPhone application (action sheet, navigation bar, etc.) with a new navigation model, called the wheel.

The wheel consists of the central object, which is the focus of the wheel, and four different Sectors around it (See Figure 2(a)). Each Sector groups objects related with the central one by particular relations. These objects are the result of a Service Logic query (see Section 3) and the relations are those inferred by the system (as described in Section 5). For instance, a Sector labeled Territory will collect all the objects that are connected to the focus of the wheel with a relation having to do with the territory; e.g. places where a product grows, shops where a product is sold, production companies where it is produced. Due to the heterogeneity of the domain items we deal with, different sector configurations 8 are possible. In particular, the sector configuration used depends on the kind of the object in the focus, and changes dynamically as the central object changes.

Using the wheel a user can browse and discover the items of the domain in a gradual and non-confusing way. Starting from the wheel of an object, the user can select a Sector, expand it and view the objects belonging to it. A Sector can contain a large number of items, due to the different possible relations between the central element and other domain elements. The user can further explore the different Sector elements, and the different relations these objects have with the object in the center by using an innovative interaction technique called "spinning the wheel", i.e. scrolling or rotating the elements in the Sector. If the user is interested in a particular item, this can become the new focus by dragging it to the center of the wheel, and the wheel configuration changes accordingly.

For example, Figure 2(a) depicts the wheel for the product Toma di Lanzo (a cheese), where the following sector configuration is used: territory (e.g. places where it is produced), people (e.g. users that tagged or voted it), cuisine (e.g. recipes using it) and products (e.g. similar products). Figure 2(b) shows the wheel where the Sector Territory is expanded. When the user changes the focus by dragging the shop El Canton dij Formagg from its Sector to the center

\footnotetext{
${ }^{8}$ We call sector configuration the name, the position and the features of the four Sectors forming the wheel.
} 


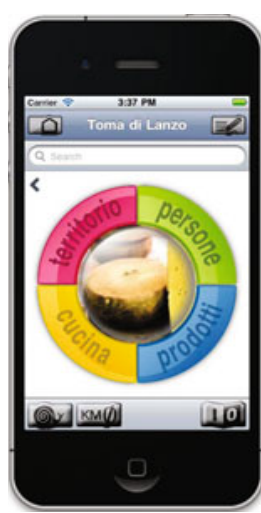

(a)

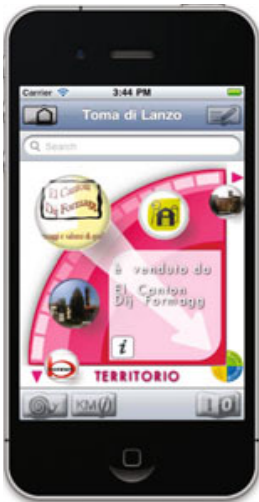

(b)

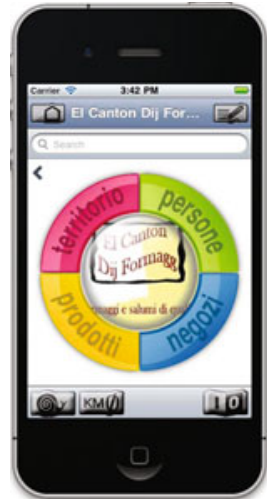

(c)

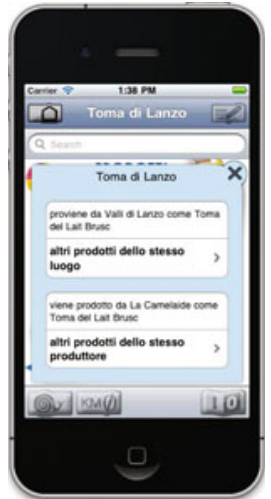

(d)

Fig. 2. Dynamic change of the sectors

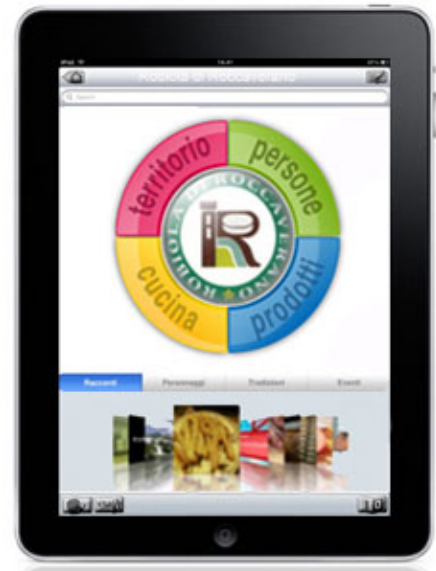

Fig. 3. Dynamic interface in video domain: iPad application

of the wheel (see Figure 2(b)), the sector configuration changes automatically: cuisine and products are replaced with products and shops (see Figure 2(c)).

If the user is not interested in a particular type of a relation or a type of an object, the interface provides different criteria to filter the items in the sectors (the buttons in the lower portion of the screen). In particular, it is possible to show only the items related to the Slow Food organization? [8], to show the items local with respect to the user position or to hide all the elements of a kind (products, producers, restaurants, etc.).

All the objects of the interface can be touched to get more details. It is also possible to investigate a particular relation in order to find out why two objects are related, i.e. which relation connects them (see Figure $2(\mathrm{~d})$ ).

\footnotetext{
${ }^{9}$ http://www.slowfood.com
} 
In addition to interaction with the wheel, users can perform various Web 2.0 actions: tagging, voting, commenting or putting into favorites the items they find interesting.

Our novel interface can be used in other multimedia contexts. We extended the WantEat iPhone application with the WantEatVideo version for Apple iPad in the video domain (see Figure 3). In this case, the videos (of the production process of certain products, for example) are connected with the objects of the wheel and change dynamically as soon as the objects in the sectors change.

\section{Evaluation}

The first proposal of our framework was tested at the early stages in order to incorporate user feedback into the final prototype. After testing the robustness of the system and the consistency of the inserted data, we performed a large scale user evaluation in October 2010 during the International Food Fair "Salone del Gusto" in Turin, Italy. More than 600 users were divided into four groups depending on their age and technology usage. They used Apple iPhones to explore the objects of our domain. We wanted to test the intuitiveness of our approach, easiness of usage and usefulness of the information obtained.

More than half of the tested users found the application easy and enjoyable to use and the information obtained useful. In addition, valuable suggestions were gathered about possible improvements and additional services (product prices availability, possibility to book a hotel/restaurant or buy a product etc.)

\section{Conclusions}

We described a novel framework for representing vast amounts of heterogeneous domain knowledge with innovative user interface and interaction technique.

The interaction experience that the application provides and the information that can be discovered is the important aspect to keep in mind. The information is presented incrementally, providing the user with non-confusing results.

The user interface was particularly designed for users in mobility, using Apple iPhone as interaction device, where we tried to introduce some novel interaction elements, while preserving many typical aspects of iPhone applications. Hence, the user has the opportunity to explore new interaction techniques and comprehensive domain knowledge, while at the same time being relaxed in the familiar environment. These new interaction metaphors have the potential of being employed in other similar applications.

Our framework can be applied to broad spectrum of different domains, reusing the approach to knowledge representation and organization, as well as a way of inferring relations between objects and people, and people themselves. One immediate future research direction that comes to mind is using a sort of similarity measure (see [7]) to asses how similar the objects in the ontology are. Creating a so called "Mixed social network" where real and virtual counterparts of objects 
and people can interact at any time is a powerful concept, influence of which should not be underestimated. It is a concept that offers many directions for future enhancements and enlargements.

\section{References}

1. Biamino, G., Grillo, P., Lombardi, I., Marcengo, A., Rapp, A., Simeoni, R., Vernero, F.: "The Wheel": an innovative visual model for interacting with a social web of things. In: Workshop on Visual Interfaces to the Social and Semantic Web, VISSW 2011 (part of IUI 2011), Palo Alto, CA, USA (2011) (accepted for publication)

2. Bizer, C., Seaborne, A.: D2RQ - Treating Non-RDF Databases as Virtual RDF Graphs. In: 3rd International Semantic Web Conference, ISWC 2004 (Poster), http://www4.wiwiss.fu-berlin.de/bizer/d2rq/

3. Catarci, T., Dongilli, P., Mascio, T.D., Franconi, E., Santucci, G., Tessaris, S.: An ontology based visual tool for query formulation support. In: Chung, S. (ed.) OTM-WS 2003. LNCS, vol. 2889, pp. 32-33. Springer, Heidelberg (2003)

4. Feldman, A., Tapia, E.M., Sadi, S., Maes, P., Schmandt, C.: ReachMedia: On-themove interaction with everyday objects. In: 9th IEEE International Symposium on Wearable Computers, ISWC 2005, pp. 52-59 (2005)

5. Franconi, E., Guagliardo, P., Trevisan, M.: An intelligent query interface based on ontology navigation. In: Workshop on Visual Interfaces to the Social and Semantic Web, VISSW 2010 (2010)

6. Guinard, D., Trifa, V., Mattern, F., Wilde, E.: From the Internet of Things to the Web of Things: Resource Oriented Architecture and Best Practices. In: Architecting the Internet of Things. Springer, Heidelberg (2011)

7. Likavec, S., Lombardi, I., Nantiat, A., Picardi, C., Theseider Dupré, D.: Threading facts into a collective narrative world. In: Aylett, R., Lim, M.Y., Louchart, S., Petta, P., Riedl, M. (eds.) ICIDS 2010. LNCS, vol. 6432, pp. 86-97. Springer, Heidelberg (2010)

8. Petrini, C.: Slow Food Nation: Why Our Food Should Be Good, Clean, and Fair. Rizzoli (2007)

9. PIEMONTE Team. WantEat: interacting with social networks of intelligent things and people in the world of enogastronomy. In: Interacting with Smart Objects Workshop 2011 (part of IUI 2011), Palo Alto, CA, USA (2011) (accepted for publication)

10. Richardson, L., Ruby, S.: RESTful web services. In: Media, O, ed. (2007), http://www4.wiwiss.fu-berlin.de/bizer/d2rq/

11. Rukzio, E., Wetzstein, S., Schmidt, A.: A Framework for Mobile Interactions with the Physical World. In: Invited Paper Special Session "Simplification of User Access to Ubiquitous ICT Services" in Wireless Personal Multimedia Communication, WPMC 2005 (2005)

12. Sonntag, D., Heim, P.: Semantic graph visualisation for mobile semantic web interfaces. In: Hertzberg, J., Beetz, M., Englert, R. (eds.) KI 2007. LNCS (LNAI), vol. 4667, pp. 506-509. Springer, Heidelberg (2007)

13. Wahlster, W.: SmartWeb: Mobile Applications of the Semantic Web. In: GI Jahrestagung (1). LNI, vol. 50, pp. 26-27. GI (2004) 